\title{
Antibiótico e Arte: uma Proposta Inovadora em Educação Médica
}

\section{Antibiotic and Art: an Innovative Approach in Medical Education}

\author{
Lara Gurgel Fernandes Távora ${ }^{\mathrm{I}}$ iD \\ Laila Maria Teixeira Amorim ${ }^{1}$ (D) \\ Ranna Jorge de Araújo ${ }^{\mathrm{I}} \mathbb{D}$ \\ Monya Garcia Baracho ${ }^{1}$ (D) \\ Mariana Pitombeira Libório ${ }^{\mathrm{I}}$
}

\section{PALAVRAS-CHAVE}

- Metodologia Ativa.

- Arte.

- Educação Médica.

\section{RESUMO}

Introdução: Com o intuito de estimular o exercício do pensamento crítico e da criatividade e considerando a complexidade vinculada ao aprendizado de antibióticos, criou-se o Teste de Sensibilidade artística aos Antimicrobianos (TSaA). Trata-se de uma atividade inovadora no ensino de antimicrobianos que avalia conhecimento e raciocínio clínico por meio de apresentações envolvendo a arte. Desde o segundo semestre de 2014, o TSaA mostrou-se uma prática integrativa no ensino de antimicrobianos para estudantes de Medicina do quinto semestre da Universidade de Fortaleza (Unifor). O objetivo deste estudo foi analisar o TSaA como metodologia de ensino/aprendizagem sobre antimicrobianos e a satisfação dos alunos com essa atividade. Métodos: Os alunos do quinto semestre do curso de Medicina foram divididos em oito grupos de 12 alunos. Os alunos receberam o desafio de utilizar a arte como ferramenta de comunicação para mostrar os seus conhecimentos sobre antimicrobianos. Cada grupo ficou responsável por apresentar conteúdos relacionados a uma classe de antimicrobiano previamente sorteada. A apresentação da atividade foi avaliada por dois docentes simultaneamente que utilizaram um instrumento específico para esse fim. Ao término do TSaA, os alunos foram convidados a responder a um questionário que abordava questões sobre a sua percepção acerca da atividade, os benefícios no contexto da aprendizagem e as dificuldades identificadas. As perguntas eram tanto objetivas quanto abertas. Resultados: Participaram do estudo 92 estudantes. A metodologia adotada para a atividade foi considerada adequada para $82 \%$ dos participantes. As formas de apresentação mais utilizadas foram encenação e música. A maioria dos participantes percebeu o TSaA como uma estratégia valiosa de facilitação de aprendizagem sobre antimicrobianos. A associação do estudo do antibiótico com a arte foi considerada uma forma divertida de aprendizagem do tema, que os levou a trabalhar de forma integrada. Na opinião dos alunos, houve realmente uma maior fixação de conteúdo com essa atividade. Conclusão: O TSaA facilitou a aprendizagem sobre antimicrobianos e estimulou a criatividade e o raciocínio crítico/lógico nos estudantes de Medicina do quinto semestre. 


\section{KEYWORDS}

- Active Methodology.

- Art.

- Medical Education.

Recebido em 13/11/19

Aceito em 2/12/19

Introdução

A aprendizagem baseada em problemas (problem based learning - PBL) é uma abordagem centrada no estudante que busca estimular o raciocínio clínico e o pensamento crítico ${ }^{1}$. Embora a PBL já fosse utilizada em outras áreas, somente em 1969 ela passou a ser aplicada como uma estratégia de ensino-aprendizagem na Faculdade de Medicina da Universidade de McMaster, no Canadá ${ }^{2}$. Desde então, várias outras estratégias de ensino centradas no estudante passaram a ser utilizadas. A team-based learning (TBL), a resolução de quiz, o simulation-based learning (SBL) e o uso de dramatização são alguns exemplos de métodos inovadores utilizados como estratégias de ensino ${ }^{3-7}$. A busca de novas estratégias de ensino e de formas inovadoras de busca ativa de conhecimento passou a ser uma constante no novo modelo de ensino médico. Procura-se, assim, ultrapassar a divisão disciplinar clássica do conhecimento científico, puramente teórico, para trabalhar a interdisciplinaridade e a participação ativa do estudante ${ }^{5}$. Nesse contexto, a dramatização, também conhecida como técnica de role-play, foi descrita como uma estratégia que favorece o desenvolvimento do raciocínio clínico dos estudantes de Medicina nos cenários clínicos e estimula a interdisciplinaridade, a criatividade e as habilidades de comunicação ${ }^{7}$. $\mathrm{O}$ uso da arte tem sido expandido para domínios além do conhecimento técnico/científico, englobando também os domínios da humanização e da ética com o paciente ${ }^{8}$.

$\mathrm{Na}$ Universidade de Fortaleza (Unifor), desde a implantação do curso de Medicina, em 2006, utiliza-se um currículo baseado em metodologias ativas, tendo a PBL como ponto central do processo de ensino-aprendizagem. A busca por métodos inovadores que estimulem e facilitem a aprendizagem é uma constante entre os docentes que fazem parte do curso. Dessa forma, pensando na complexidade vinculada ao aprendizado de antibióticos, criou-se uma atividade denominada Teste

\section{ABSTRACT}

Introduction: To stimulate the practice of critical thinking and creativity and considering the complexity associated with antibiotic learning, the Antimicrobial Artistic Sensitivity Test (TSaA) was created, which is an innovative activity in antimicrobial teaching that evaluates knowledge and clinical reasoning through artistic presentations. Since the second semester of 2014 (2014.2), TSaA has proven to be an integrative practice in antimicrobial teaching for fifth-semester medical students of the University of Fortaleza/CE (UNIFOR). The objectives of this study were to analyze TSaA as a methodology of antimicrobial teaching / learning and students' satisfaction with this practice. Methods: Fifth-semester medical students were divided into eight groups, each one with twelve participants. Students were challenged to show their knowledge of one class of antimicrobial drug using art as the communication tool. Each group was assigned to work with a specific class of antibiotic, which was previously randomly selected. Two teachers simultaneously evaluated the presentation, using a specific form to carry out this evaluation. In the end of the activity, students were invited to answer a questionnaire, with ojective and subjective questions, addressing their perceptions of the activity, the benefits on antibiotic learning music were the most frequently chosen forms of presentation. The majority of the participants indicated TSaA as a valuable strategy to facilitate antimicrobial learning. The association between antibiotic study and art was considered an amusing way of content learning. According to students' perception, this practice also stimulated teamwork. In the students' opinion, there was indeed a greater content learning with this activity. Conclusion: The Antimicrobial Artistic Sensitivity Test facilitated antimicrobial learning, stimulating the creativity and critical/logical thinking of fifth-semester medical students. 
peso 4, e a organização/o trabalho de equipe, peso 2. Essa atividade era opcional e acontecia após ao final do semestre, uma semana após todo o conteúdo de antimicrobianos ter sido dado em conferências.

\section{METODOLOGIA}

Trata-se de um estudo retrospectivo, transversal, de abordagem mista, qualitativa e quantitativa. A pesquisa foi desenvolvida no curso de Medicina da Unifor, com alunos do quinto semestre (período do curso em que se aborda o conteúdo sobre antimicrobianos). Incluíram-se alunos que cursaram o quinto semestre no segundo semestre de 2015.

Os alunos foram convidados a responder a um questionário logo após o término da atividade do TSaA. Nesse questionário, havia perguntas (objetivas e abertas) sobre a percepção dos alunos acerca da atividade, os seus benefícios no contexto da aprendizagem e as dificuldades identificadas (Figura 1).

$\mathrm{Na}$ análise estatística, utilizou-se o programa SPSS 16.0, e calcularamse as medidas de frequência, tendência central e análise de conteúdo para as variáveis subjetivas. A análise das questões abertas incluíram a categorização, identificação de frequências e seleção de exemplos de relatos mais ilustrativos das categorias.

O estudo foi aprovado pelo Comitê de Ética em Pesquisa da Unifor (Parecer $\mathrm{n}^{\circ}$ 1.346.0440).

\section{RESULTADOS}

Dos 96 alunos matriculados no semestre, foram incluídos 92 alunos no estudo. A maioria era do sexo masculino (51\%), e a média de idade, de 22,4 anos (+/- 3 anos). A maioria utilizou como fonte de estudo para a elaboração da atividade livros específicos sobre o assunto (67\%). A minoria $(9,9 \%)$ buscou periódicos científicos ou vídeos na internet. Apenas um grupo não conseguiu atingir o peso máximo na avaliação do conteúdo, tendo atingido peso 3.

As formas de apresentação mais escolhidas pelos alunos foram encenação e música. Alguns grupos utilizaram ainda formas mistas de apresentação (Gráfico 1).

\section{Figura 1}

Percepção dos alunos do quinto semestre do curso de Medicina da Unifor sobre o Teste de Sensibilidade artística aos Antimicrobianos (TSaA) instrumento de coleta de dados

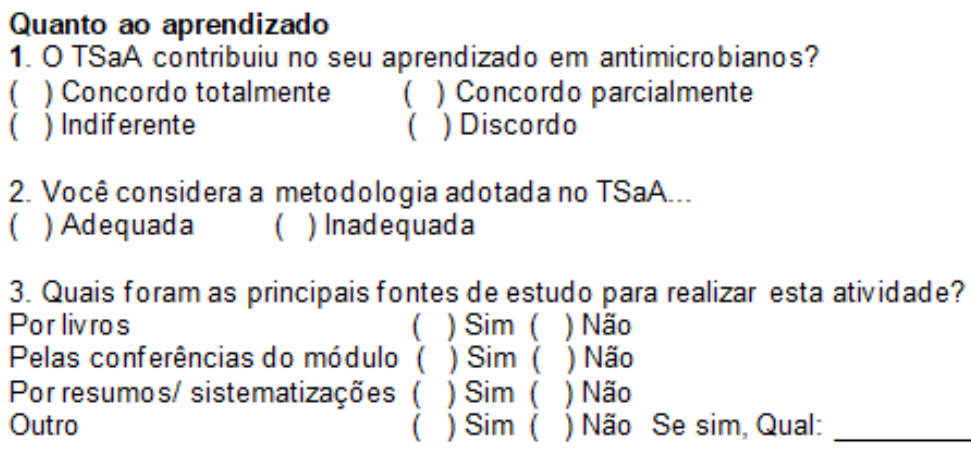

5. Qual foi sua principal forma de apresentação?

( ) Encenação na hora da apresentação ou gravada em vídeo

( ) Música

( ) Cordel

( ) Poema/poesia

( ) Outro Qual:

6. Conseguiu que sua apresentação abordasse os principais pontos? (Mecanismos de ação, espectro de ação, mecanismo de resistência e ef eitos adversos).

( ) Sim, todos. ( ) Quase todos ( ) Poucos ( ) Não consegui realizar

7. Você sentiu dificuldade para a realização da atividade?

( ) Não ( ) Sim Se sim, qual?

8. Houve contribuição de todos em sua equipe?

( ) Todos contribuíram ( ) Quase todos contribuíram ( )Poucos contribuíram

Pontos fortes e fracos:

9. Quais os principais benefícios e pontos fortes desta atividade?

10. Quais as principais dificuldades e pontos fracos desta atividade? 


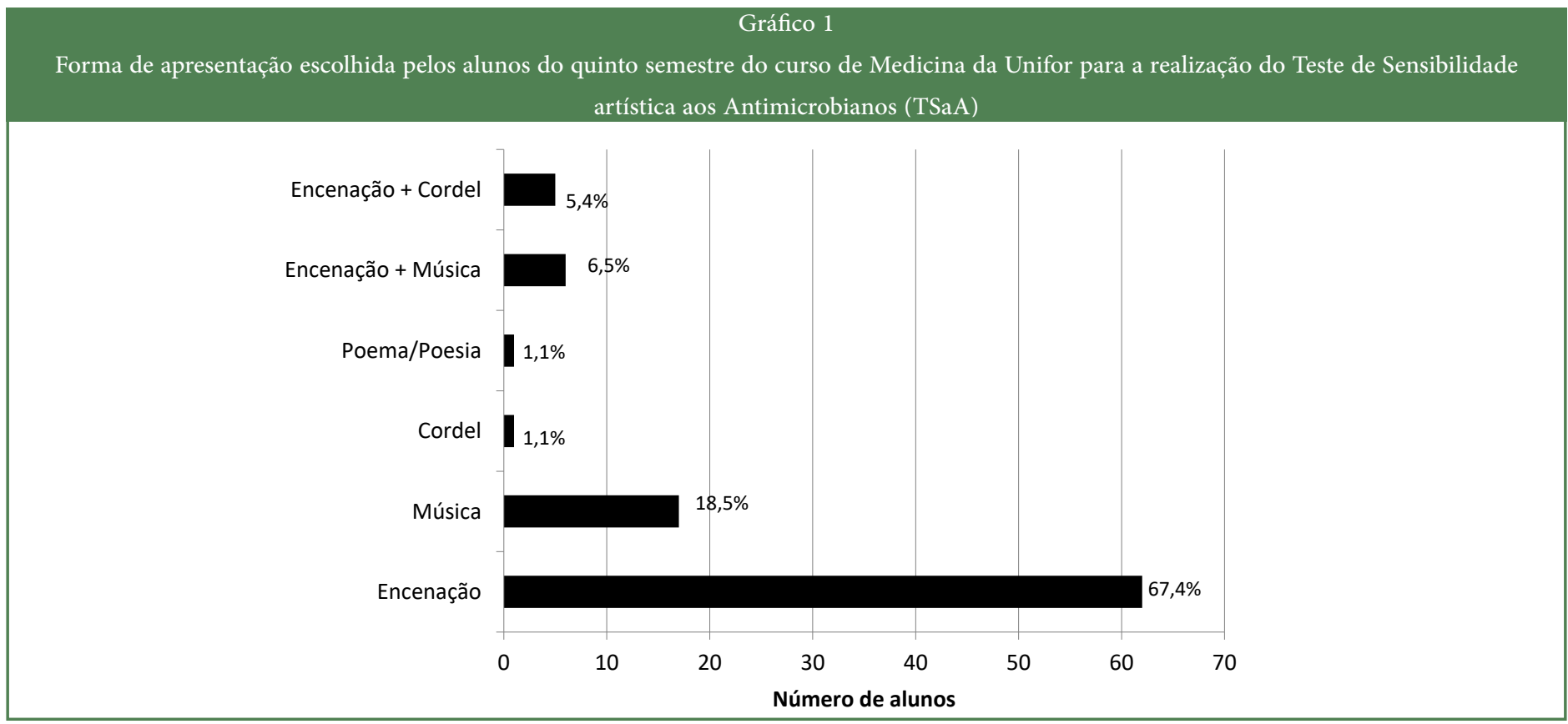

Fonte: Elaborado pelos autores.

Para $82 \%$ dos alunos, a metodologia adotada na atividade foi adequada. Quando perguntados se a participação na atividade facilitou o aprendizado sobre antimicrobianos, a maioria (51\%) concordou totalmente e $44 \%$ concordou parcialmente. Apenas um aluno discordou. Uma pequena parcela dos alunos relatou que não conseguiu abordar totalmente o conteúdo exigido, embora tenha conseguido abordar a maioria dele $(6,5 \%)$.

Vinte e cinco alunos $(27,1 \%)$ responderam que encontraram alguma dificuldade na execução da atividade. As dificuldades relatadas pelos participantes foram relacionadas principalmente ao tempo para a elaboração da atividade e ao conteúdo. Para oito alunos (8,7\%), o tempo para elaboração foi insuficiente para que fosse possível organizar e elaborar melhor a atividade. Sobre o conteúdo, 15 participantes (16,3\%) relataram que tiveram dificuldade na apresentação do tema sorteado de forma lúdica. Eis duas respostas que ilustram bem essa percepção: "Difícil abordar de forma lúdica temas complexos" e "Adaptar conteúdo científico tão extenso à arte da encenação".

Cinco alunos $(5,4 \%)$ referiram ainda dificuldade em decorar as letras das músicas e/ou o texto das encenações e organizar o trabalho em equipe. Apesar de a dificuldade com a organização da equipe ter sido citada, quando perguntados se houve colaboração de todos os membros da equipe, 85 alunos $(92,4 \%)$ responderam positivamente.

Quando questionados sobre os pontos fortes da atividade, os aspectos mais citados pelos alunos foram a facilitação/fixação do aprendizado $(48,9 \%)$ e a associação de humor/diversão com o aprendizado $(27,1 \%)$. Essas percepções ficaram bem ilustradas em algumas das respostas deles:

“De forma engraçada e leve conseguimos abordar um conteúdo tão denso nos seus pontos principais" e "O TSaA foi uma forma de facilitação de aprendizagem mas com um aprendizado divertido".

Também foram mencionadas outras questões, como a união e integração do grupo e a metodologia ativa, como pontos marcantes da atividade. Para um aluno o ponto forte foi "A forma divertida de aprendizado com a valorização de todos que ajudaram".

Alguns pontos fracos foram apontados por 31 alunos. Muitos mencionaram como fatores limitantes a falta de tempo suficiente para preparar a atividade $(15,2 \%)$ e o cansaço $(4,3 \%)$. Algumas das respostas ilustram bem essa percepção: "O cansaço do final do semestre e o pouco tempo para preparação" e "Pouco tempo para organizar a atividade porque ainda temos que estudar para a prova né...”.

\section{Discussão e Conclusão}

A facilitação da aprendizagem com o TSaA foi uma percepção comum para a maioria dos alunos, sendo inclusive citado por muitos como um dos pontos fortes da atividade. A maioria dos grupos optou pela dramatização como forma de comunicação do conteúdo. Dolev, Krohner e Braverman', em estudo realizado na Faculdade de Medicina da Universidade de Yale, constataram que a utilização da arte como ferramenta de aprendizagem foi eficaz, melhorando a capacidade de diagnóstico clínico dos estudantes quando comparados a alunos do mesmo semestre que receberam apenas aulas teóricas e práticas habituais. A contribuição da aprendizagem por meio da arte para o estímulo/desenvolvimento do pensamento crítico também foi apontada por outros autores ${ }^{10}$. $\mathrm{O}$ uso da simulação por meio de dramatização, mesmo quando o aluno é apenas um observador, parece ser um método eficaz de aprendizado de conteúdo ${ }^{11,12}$. Ainda, alguns estudos demonstraram que o uso de dramatização como ferramenta de aprendizagem resultou em uma maior fixação de conteúdo quando comparado até mesmo com outros recursos audiovisuais, como vídeos ${ }^{13}$.

Apesar de a organização do trabalho de equipe ter sido citada por alguns como uma das dificuldades do TSaA, 92\% dos alunos relataram uma participação plena dos componentes do grupo na realização da atividade, e muitos apontaram a integração e a união do grupo como um dos pontos fortes dessa metodologia. A utilização de atividades que estimulem o trabalho em equipe nem sempre foi uma preocupação na área da educação médica. O’Connell e Pascoe ${ }^{14}$ mostraram que, até o início 
dos anos 2000, a literatura científica sobre a importância do trabalho de equipe na formação médica era bastante escassa. As mudanças na prática médica e a necessidade cada vez maior de profissionais da saúde capazes de trabalhar de forma interdisciplinar estimularam o surgimento de novas estratégias na docência. Muitas dessas estratégias têm o trabalho de equipe, o estímulo à atividade colaborativa e à comunicação interpares como focos centrais ${ }^{15}$. Vale ressaltar ainda que, atualmente, a interconectividade é uma realidade entre os estudantes de Medicina, o que acaba por facilitar práticas que estimulem o trabalho colaborativo e de equipe ${ }^{16}$. Entretanto, vários são os tipos de conflito que podem ocorrer durante a elaboração/ execução de trabalhos em equipe, como aqueles referentes à distribuição de tarefas, a processos ou relacionamentos. $\mathrm{Na}$ educação médica, os conflitos intragrupos devem ser trabalhados com cautela ${ }^{17}$. Nesse contexto, a utilização de estratégias que promovam o desenvolvimento de trabalho cooperativo, em detrimento de atividades que estimulem a competição interpares, torna-se essencial para que se possa garantir uma atmosfera de aprendizado individual e crescimento de grupo ${ }^{18}$.

O excesso de trabalho e o tempo exíguo para realização das atividades acadêmicas já foram apontados por alguns autores como fatores de estresse e desencadeadores de burnout em alguns estudantes de Medicina ${ }^{19}$. No presente estudo, uma das dificuldades mais citadas pelos participantes foi o pouco tempo para a organização da atividade. Esse resultado aponta para a necessidade de uma melhor programação do cronograma de realização da atividade para que possamos atingir o maior rendimento possível dos alunos, sem o ônus de estresse desnecessário.

Outra dificuldade relatada por seis alunos $(6,5 \%)$ foi não conseguir abordar completamente o conteúdo exigido. Alguns relacionaram essa dificuldade à complexidade do assunto. Entendemos que essa dificuldade pode ser uma das limitações dessa metodologia. Entretanto, acreditamos que o baixo percentual de alunos com essa dificuldade e o fato de todos pertencerem ao mesmo grupo sugerem que qualquer obstáculo surgido teve uma importância menor no desenvolvimento da atividade de forma geral e que, possivelmente, estaria mais relacionado a uma dificuldade de organização pontual desse grupo específico.

Conclui-se que o TSaA teve um alto grau de aceitação entre os alunos e entra como forma complementar de ensino/aprendizagem, de modo a facilitar a fixação do conteúdo e incentivar a criatividade nas apresentações, o trabalho em grupo e o pensamento crítico, características fundamentais no ensino médico. Entretanto, faz-se necessário repensar o tempo disponibilizado para a elaboração da atividade para que o aproveitamento seja ainda maior.

\section{REFERÊNCIAS}

1. Neville AJ. Problem-based learning and medical education forty years on a review of this effects on knowledge and clinical performance. Medical Principles and Practice 2009;18:1-9.

2. Borges MC, Chachá SGF, Quintana SM, Freitas LCC, Rodrigues MLV. Aprendizado baseado em problemas. Medicina (Ribeirão Preto) 2014;47(3):301-7.

3. Michaelsen LK, Sweet M. The essential elements of team-based learning. New Directions for Teaching and Learning 2008;116:7-27.

4. Thompson BM, Schneider VF, Haidet P, Levine RE, McMahon KK, Perkowski LC et al. Team-based learning at ten medical schools: two years later. Med. educ. 2007;41(3):250-7.

5. Devi, K. Quiz as an innovative approach in teaching community medicine to medical students. National Journal of Community Medicine 2014;5(2):182-5.

6. Abdulmohsen HAE. Simulation-based medical teaching and learning. J Family Community Med 2010;17(1):35-40.

7. Rabelo L, Garcia VL. Role-play for the development of communication skills. Rev. bras. educ. méd. 2015;39(4):586-96.

8. Amorim KPC, Rocha AKC, Silva ICS, Melo LMB, Araújo MAA. Mediarte com amor e humor: uma experiência a partir do olhar dos participantes. Rev. bras. educ. méd. 2015;39(2):294-301.

9. Dolev JC, Krohner L, Braverman IM. Using fine art to enhance visual diagnostic skills. JAMA 2001;286:1020-1.

10. Chan, ZC. Exploring creativity and critical thinking in traditional and innovative problem-based learning groups. J. clin. nurs. 2013;22:2298-307.

11. Roberts D. Vicarious learning: a review of the literature. Nurse educ. pract. 2010;10:13-6.

12. O'Regan S, Molloy E, Watterson L, Nestel D. Observer roles that optimize learning in healthcare simulation education: a systematic review. Advances in Simulation 2016;1:4. doi: 10.1186/s41077-015-0004-8.

13. Robles MJ, Miralles R, Esperanza A, Riera M. Different ways to present clinical cases in a classroom: video projection versus live representation of a simulated clinical scene with actors. BMC med. educ. 2019;19:70. doi: 10.1186/s12909-019-1494-1.

14. O'Connell MT, Pascoe JM. Undergraduate medical education for the 21th century: leadership and teamwork. Fam. med. 2004;3:S51-S56.

15. Parmelee D, Michaelsen LK, Cook S, Hudes PD. Team-based learning: a practical guide: AMEE guide $\mathrm{n}^{\circ}$ 65. Med. teach. 2012;34:e275-e287.

16. Borges NJ, Manuel RS, Elam CL, Jones BJ. Differences in motives between Millennial and Generation X medical students. Med. educ. 2010;44(6):570-6.

17. Greer LL, Saygi O, Aaldering H, de Dreu CKW. Conflict in medical teams: opportunity or danger? Med. educ. 2012;46(10):935-42.

18. Mafinejad MK, Ahmady S, Arabshahi SKS, Bigdeli S. Interprofessional education in the integrated medical education and health care system: a contente analysis. J Adv Med Educ Prof 2016;4(3):103-10.

19. Dyrbye LN, Harper W, Durning SJ, Moutier C, Thomas MR, Massie FS Jr. et al. Patterns of distress in US medical students. Med. teach. 2011;33(10):834-9.

\section{CONTRIBUIÇÃO DAS AUTORAS}

Lara Gurgel Fernandes Távora e Mariana Pitombeira Libório: professoras orientadoras.

Laila Maria Teixeira Amorim, Ranna Jorge de Araújo e Monya Garcia Baracho: pesquisadoras discentes que realizaram a coleta, análise dos dados e escrita inicial do artigo.

\section{CONFLITO DE INTERESSES}

As autoras declaram não haver conflito de interesses neste estudo. 


\section{ENDEREÇO PARA CORRESPONDÊNCIA}

Lara Gurgel Fernandes Távora

Avenida Engenheiro Santana Junior, 2947, apt. 1402, Bairro Cocó.

Fortaleza/CE. CEP 60192205.

E-mail: laratavora@gmail.com and reproduction in any medium, provided the original work is properly cited.

\begin{tabular}{l|l}
\hline REVISTA BRASILEIRA DE EDUCAÇÃO MÉDICA \\
\hline $44(1): \mathrm{e} 035 ; 2020$
\end{tabular}

